\title{
Gastrectomía extendida en cáncer gástrico. Evaluación de la morbilidad y mortalidad postoperatoria
}

\author{
JEAN M. BUTTE ${ }^{1}$, NICOLÁS KERRIGAN ${ }^{1}$, ENRIQUE WAUGH ${ }^{1}$, \\ MANUEL MENESES ${ }^{1}$, HUGO PARADA ${ }^{1}$, ÁLVARO VISSCHER ${ }^{2 a}$, \\ FEDERICO BECKER ${ }^{2 a}$, HERNÁN DE LA FUENTE ${ }^{1}$
}

\section{Complications and mortality of extended gastrectomy for gastric cancer}

Background: Extended gastrectomy allows a complete tumor excision in patients with advanced gastric cancer. Aim: To compare the surgical results of extended (ER) and non-extended gastrectomy (NER) among patients with gastric cancer, and determine factors associated with complications and mortality. Material and Methods: Review of medical records of patients with gastric cancer who underwent complete resection between 2002 and 2008 in an oncological hospital. Demographics, patient-related and therapeutic features were compared between groups, and independent factors were established with multivariate analysis. Results: Seventy four patients, (44 men, median age 62 years) underwent an ER and 103 patients, (56 men, median age 61 years) a NER. Specifically, ER included splenectomy alone in 27 patients, splenectomy associated with other procedure in 24, partial esophagectomy in 18, distal pancreatectomy in 13, hemicolectomy in 8, total esophagectomy in 7 , partial hepatectomy in 4, and adrenalectomy in 1. Postoperative complications were observed in 19 patients treated with an ER (26\%) and in 11 patients treated with a NER (11\%), $p<0.05$. Serious complications were higher in patients who underwent an ER compared with NER (6 patients (8\%) vs. $4(4 \%)$, respectively) $p<0.05$. In the same way, mortality was higher in patients treated with ER when it was compared with NER (4 patients (5\%) vs two (2\%), respectively), $p<0.05$. ER and serum albumin levels were independent factors associated to a higher risk of mortality and rate of complications. Conclusions: ER was associated with a higher rate of general and severe complications, and mortality.

(Rev Med Chile 2010; 138: 1487-1494).

Key words: Gastrectomy; Stomach neoplasms; Survival rate.

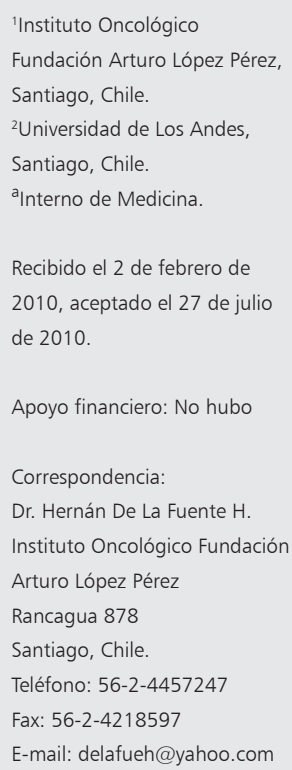

1 1 cáncer gástrico tiene una alta incidencia en el mundo y es la segunda causa de muerte por neoplasia maligna ${ }^{1}$. Lamentablemente, a pesar de los avances importantes en el diagnóstico y tratamiento, la sobrevida a 5 años es pobre, porque está determinada fundamentalmente por el diagnóstico tardío que tienen la mayoría de los enfermos ${ }^{2}$. En Chile, es la primera causa de muerte por cáncer, el número de fallecidos es cercana a la mortalidad observada en enfermedades crónicas prevalentes ${ }^{3}$, más de $80 \%$ de los pacientes se presentan en etapas avanzadas y $50 \%$ de ellos tienen compromiso de órganos vecinos 4 .

Está claramente demostrado que cuando la enfermedad está localizada, el tratamiento de elección es la resección quirúrgica ${ }^{5}$. Estudios recientes 
han confirmado que esto también sería aplicable a aquellos pacientes con tumores localmente avanzados con infiltración de órganos adyacentes ${ }^{6,7}$. A pesar de esto, la invasión local sería otro factor de mal pronóstico, independiente del tratamiento quirúrgico radical $\mathrm{R} 0^{8}$. Sin embargo, esto sólo se puede demostrar con certeza después de la extracción y análisis completo de la pieza quirúrgica9.

La gastrectomía extendida (GE) o multivisceral, se define como la extirpación de los sitios de extensión tumoral extragástrica en contigüidad con la resección gástrica asociado a disección ganglionar y su principal objetivo es lograr una resección completa con márgenes negativos ${ }^{10}$. Debe diferenciarse de la gastrectomía paliativa, cuyo objetivo es controlar aquellos síntomas que están afectando la calidad de vida, como la obstrucción o el sangrado, en enfermos en que no se estima una larga sobrevida ${ }^{11}$. La GE es considerada una cirugía de mayor envergadura, asociada a una mayor morbilidad y mortalidad perioperatoria, la mayoría de las veces relacionada con reconstrucciones digestivas adicionales ${ }^{12}$. Su beneficio clínico es controversial, y debe analizarse balanceando factores de corto plazo, como la morbilidad y mortalidad perioperatoria con objetivos a largo plazo, como la calidad de vida, sobrevida libre de enfermedad y sobrevida global de los pacientes ${ }^{13}$.

Los objetivos de este estudio son evaluar la morbilidad y mortalidad de los pacientes tratados con una GE, compararla con los tratados con una gastrectomía no extendida (GNE) y determinar factores asociados a morbilidad y mortalidad.

\section{Material y Métodos}

\section{Pacientes}

Este es un estudio retrospectivo a partir de los registros clínicos de los pacientes con diagnóstico de cáncer gástrico resecado en el Instituto Oncológico Fundación Arturo López Pérez entre junio de 2000 y junio de 2008. Los datos fueron recolectados en forma retrospectiva hasta junio de 2007 y en forma prospectiva desde esa fecha hasta el cierre del estudio. Se analizaron características demográficas (edad y género), el tratamiento quirúrgico y las complicaciones derivadas de este. Los pacientes fueron divididos en grupo A, pacientes tratados con una GE y grupo B, pacientes tratados con una GNE.
Todos los enfermos fueron estudiados y tratados de acuerdo al mismo protocolo quirúrgico, que ha sido previamente comunicado ${ }^{14}$.

\section{Tratamiento quirúrgico administrado}

El día previo a la operación se realizó una preparación intestinal con polietilenglicol. Luego de descartar la presencia de enfermedad metastásica en el abdomen, se evaluó la ubicación tumoral, y se decidió el tipo de tratamiento quirúrgico así como la forma de reconstrucción del tránsito intestinal. Se practicó una gastrectomía total a aquellos enfermos con un tumor localizado en el tercio superior o medio del estómago y una gastrectomía subtotal a aquellos enfermos con un tumor localizado en el tercio inferior del estómago.

La gastrectomía estándar (GNE) incluyó el omento mayor y menor asociado a disección ganglionar D2, sin esplenectomía ni pancreatectomía de rutina. Por otra parte, gastrectomía extendida fue definida como aquella operación que incluyó la resección gástrica estándar y al menos uno de los siguientes órganos: esófago, bazo, colon, páncreas, diafragma, glándula suprarrenal o hígado. La decisión de ampliar la resección gástrica estándar fue tomada por el cirujano, de acuerdo a los hallazgos de la exploración intraoperatoria y su objetivo fue resecar el tumor primario asi como todos los sitios de posible extensión tumoral directa necesarios para lograr una resección R0.

La anastomosis esófago-yeyunal se realizó término-lateral, con una engrapadora circular $\mathrm{N}^{\circ} 21$ ó 25, de acuerdo a las características del paciente y decisión del cirujano. La anastomosis se cubrió con un segundo plano seroso-muscular con monocryl ${ }^{\circledR} 4-0$ continuo. En los pacientes en que se agregó una esofagectomía a la resección, la anastomosis esófago-colónica cervical se realizó con monocryl ${ }^{\circledR}$ 4-0 en forma continua. Todas las otras anastomosis abdominales se realizaron en forma manual con monocryl ${ }^{\circledR}$ 4-0 en forma continua. En todos los enfermos se realizó una colecistectomía y una apendicectomía cuando estos órganos estuvieron presentes.

\section{Complicaciones postoperatorias}

Todas las complicaciones postoperatorias fueron analizadas y estratificadas de acuerdo a su gravedad $^{13}$. Se consideró complicación postoperatoria a aquella que ocurrió hasta 60 días de la operación. Se consideró mortalidad postoperatoria a la que 
ocurrió hasta 60 días de la operación y se asoció a la cirugía.

No se realizó un estudio contrastado para evaluar la presencia de una fístula de la anastomosis esofagoyeyunal en forma rutinaria. Este examen sólo fue realizado en un grupo seleccionado de pacientes que durante la evolución postoperatoria presentaron algún signo clínico que sugirió la presencia de alguna complicación. Específicamente, se realizó un estudio contrastado con solución hidrosoluble (no baritada) para evaluar la anastomosis propiamente tal y una tomografía computada de tórax, abdomen y pelvis para evaluar la presencia de alguna colección. El tratamiento específico de una fístula en general fue expectante e incluyó el manejo medico general (reposo intestinal, soporte nutricional y antibioticoterapia). Aquellos pacientes con un cuadro séptico asociado a una colección imposible de drenar en forma completa por vía percutánea fueron tratados en forma quirúrgica.

\section{Estudio anatomopatológico}

En todos los enfermos, el número y nivel de las metástasis ganglionares fueron determinadas de acuerdo a la clasificación japonesa de cáncer gástrico ${ }^{15}$. Todos los enfermos fueron etapificados de acuerdo a la clasificación TNM-American Joint Committee on Cancer (AJCC) para cáncer gástrico del año $2002^{16}$. Se definió resección con intención curativa o R0 a aquella operación en la que no se observó compromiso tumoral microscópico de los márgenes quirúrgicos luego del estudio anatomopatológico definitivo.

\section{Análisis estadístico}

Todos los pacientes fueron seguidos hasta el término del estudio o su muerte.

En el análisis estadístico se usó t de Student, Mann-Whitney, $\chi^{2}$ o test de Fisher. Se realizó un análisis bivariado, y un análisis multivariado con el método de Cox. Se consideró un valor de $\mathrm{p}<0,05$ como significativo. Con el fin de conocer la sobrevida de los enfermos sometidos a una gastrectomía extendida, se obtuvieron curvas de sobrevida con el método de Kaplan-Meier.

\section{Resultados}

Durante el período estudiado, 177 enfermos fueron sometidos a una gastrectomía con el diag- nóstico de cáncer gástrico. Fueron 101 (57\%) hombres y 76 (43\%) mujeres con una edad media de 61,5 años (rango, 25-87 años). El grupo A (GE) estuvo formado por 74 pacientes (44 hombres y 30 mujeres, edad media: 62 años) y el grupo B (GNE) estuvo conformado por 103 enfermos (56 hombres y 47 mujeres, edad media: 61 años). El estudio anatomopatológico final mostró que a 158 $(89,3 \%)$ pacientes se les realizó una resección R0, a $10(5,6 \%)$ una resección $\mathrm{R} 1$ y a $9(5,1 \%)$ una resección R2. En el grupo A, 65 (87,8\%) pacientes fueron tratados con una resección R0, $5(6,8 \%)$ con una R1 y $4(5,4 \%)$ con una R2. En el grupo B, $93(90,3 \%)$ pacientes recibieron una resección R0, 5 (4,9\%) una R1 y $5(4,9 \%)$ una R2.

En el grupo A se resecaron 107 órganos extra gástricos (1,45 órganos por paciente). Esplenectomía exclusiva en 27, esplenectomía asociada a otro procedimiento en 24 , esofagectomía parcial en 18 , pancreatectomía corporo caudal o distal en 13 , resección parcial de colon en 8 , esofagectomía total en 7 , resección parcial de diafragma en 5 , hepatectomía parcial en 4 y resección de la glándula suprarrenal izquierda en 1. A 50 pacientes se les resecó un órgano extragástrico, a 20 se le resecaron dos y a 4 se le resecaron tres.

El estudio anatomopatológico de la pared gástrica del grupo de enfermos tratados con una GE mostró compromiso de la submucosa (T1) en 2 $(2,7 \%)$ pacientes, compromiso de la capa muscular (T2a) en $6(8,1 \%)$, compromiso subseroso (T2b) en $18(24,3 \%)$ pacientes, compromiso seroso (T3) en $41(55,4 \%)$ y compromiso del órgano adyacente resecado (T4) en 7 (9,5\%). En la evaluación específica de cada órgano resecado se observó compromiso tumoral del esófago en los 25 (100\%) pacientes sometidos a una resección esofágica (parcial o total), compromiso tumoral del diafragma en los $5(100 \%)$ enfermos sometidos a una resección parcial de diafragma, compromiso tumoral del páncreas en $4(30,7 \%)$ pacientes en que se practicó una pancreatectomía distal, compromiso tumoral del colon o mesocolon transverso en $3(37,5 \%)$ pacientes sometidos a una colectomía parcial y compromiso tumoral de la glándula suprarrenal izquierda en un paciente en que se resecó parte de parte de dicho órgano. Por el contrario, no se observó compromiso tumoral del bazo en ninguno de los enfermos sometidos a una esplenectomía ni compromiso tumoral del hígado en ninguno de los pacientes tratados con una hepatectomía parcial. 
A pesar de esto, 15 (55,5\%) enfermos sometidos a una esplenectomía exclusiva, 6 (43\%) enfermos tratados con una pancreatectomía, 5 (62,5\%) pacientes sometidos a una colectomía parcial y 3 (75\%) enfermos tratados con una hepatectomía parcial presentaron compromiso tumoral de la serosa gástrica, lo que confirma el avanzado estado tumoral al momento de la resección. Sesenta y cinco $(87,8 \%)$ enfermos presentaron compromiso ganglionar. La distribución según procedimiento y etapa tumoral se observa en la Tabla 1.

Treinta (16,9\%) enfermos presentaron una o más complicaciones; $19(25,6 \%)$ en el grupo A y 11 $(10,6 \%)$ en el grupo B $(p<0,05)$. En el grupo A se observó que 11 de 50 enfermos (22\%) sometidos a la resección de un órgano extragástrico, 7 de 20 (35\%) sometidos a la resección de 2 órganos y uno de $4(25 \%)$ pacientes sometidos a la resección de tres órganos extragástricos presentaron complicaciones postoperatorias. Las complicaciones se detallan en la Tabla 2.

Cinco $(6,7 \%)$ pacientes del grupo A y $4(3,8 \%)$ del grupo $\mathrm{B}$ fueron reoperados. Las causas fueron hemoperitoneo (1 paciente), absceso subfrénico (2 pacientes) y filtración de la anastomosis esófagoyeyunal (2 pacientes) en el grupo A y hemoperitoneo (1 paciente), absceso subfrénico (1 paciente), íleo mecánico precoz (1 paciente) y filtración del muñón duodenal (1 paciente) en el grupo B. Seis $(8,2 \%)$ enfermos del grupo A y 4 (3,9\%) enfermos del grupo $\mathrm{B}$ presentaron una complicación grado 3 ó 4 (complicación más grave) $(\mathrm{p}<0,05)$. En la Tabla 3 se observan las complicaciones de acuerdo a su gravedad.

\section{Tabla 1. Distribución según etapa tumoral y procedimiento}

\begin{tabular}{|ccc|}
\hline $\begin{array}{l}\text { Etapa } \\
\text { tumoral }\end{array}$ & $\begin{array}{c}\text { GE } \\
\mathbf{n}=\mathbf{7 4}(\%)\end{array}$ & $\begin{array}{c}\text { GNE } \\
\mathbf{n}=\mathbf{1 0 3}(\%)\end{array}$ \\
\hline IA & $1(1,3 \%)$ & $20(19,4 \%)$ \\
IB & $5(6,8 \%)$ & $16(15,5 \%)$ \\
II & $13(17,6 \%)$ & $23(22,3 \%)$ \\
IIIA & $13(17,6 \%)$ & $16(15,5 \%)$ \\
IIIB & $6(8,1 \%)$ & $10(9,7 \%)$ \\
IV & $36(48,6 \%)$ & $18(17,5 \%)$ \\
\hline
\end{tabular}

Tabla 2. Complicaciones postoperatorias

\begin{tabular}{|c|c|c|}
\hline Complicación & GE & GNE \\
\hline Atelectasia & 2 & 3 \\
\hline Trombosis venosa profunda & 3 & \\
\hline Neumonía & 3 & \\
\hline Absceso subfrénico & 2 & 1 \\
\hline Filtración esófago-yeyunal & 2 & \\
\hline Tromboembolismo pulmonar & 2 & \\
\hline Infección de herida operatoria & 1 & 1 \\
\hline Hemoperitoneo & 1 & 1 \\
\hline íleo mecánico precoz & & 1 \\
\hline Filtración muñón duodenal & & 1 \\
\hline Peritonitis por Candida albicans & & 1 \\
\hline $\begin{array}{l}\text { Obstrucción intestinal por hernia } \\
\text { transhiatal aguda }\end{array}$ & 1 & \\
\hline Neumotórax & 1 & \\
\hline Accidente vascular encefálico & 1 & \\
\hline Cólico renal & 1 & \\
\hline Candidiasis sistémica & 1 & \\
\hline Derrame pleural & 1 & \\
\hline Retención urinaria aguda & 1 & \\
\hline Colitis pseudomembranosa & 1 & \\
\hline Sepsis secundaria a peritonitis & & 1 \\
\hline Arritmia & 1 & \\
\hline Insuficiencia renal aguda & 1 & \\
\hline
\end{tabular}

Tabla 3. Complicaciones según severidad

\begin{tabular}{|ccc|}
\hline $\begin{array}{c}\text { Grado de } \\
\text { complicación }\end{array}$ & $\begin{array}{c}\text { GE } \\
\mathbf{n = 7 4}\end{array}$ & $\begin{array}{c}\text { GNE } \\
\mathbf{n = 1 0 3}\end{array}$ \\
\hline 1 & $9(12,1 \%)$ & $7(6,8 \%)$ \\
\hline 2 & $5(6,8 \%)$ & $0(0 \%)$ \\
3 & $5(6,8 \%)$ & $3(2,9 \%)$ \\
4 & $1(1,4 \%)$ & $1 \quad(1 \%)$ \\
\hline
\end{tabular}

Complicación $3+4$, GE vs. GNE $(p<0,05)$. 
Gastrectomía extendida en cáncer gástrico - J.M. Butte et al

Tabla 4. Análisis bivariado. Factores asociados con morbilidad y mortalidad

\begin{tabular}{|lccc|}
\hline Variable & OR & IC 95\% & p \\
\hline Gastrectomía extendida & 3,12 & $1,46-6,69$ & 0,003 \\
\hline Sexo masculino & 2,83 & $1,24-6,46$ & 0,013 \\
\hline Edad (mayor de 65 años) & 1,03 & $1-1,07$ & 0,046 \\
\hline Recuento de linfocitos bajo lo normal & 0,99 & $0,99-1,00$ & 0,039 \\
\hline Albúmina normal vs albumina $<3,5 \mathrm{mg} / \mathrm{dl}$ & 0,33 & $0,15-0,73$ & 0,006 \\
\hline
\end{tabular}

Tabla 5. Análisis multivariado. Factores asociados con movilidad y mortalidad

\begin{tabular}{|lccc|}
\hline Variable & OR & IC $\mathbf{9 5 \%}$ & P \\
\hline Albúmina normal vs albúmina $<3,5$ & 0,35 & $0,16-0,76$ & 0,008 \\
\hline Gastrectomía extendida & 3,21 & $1,46-7,07$ & 0,004 \\
\hline
\end{tabular}

Ajustado por edad y sexo.

Hubo $6(3,4 \%)$ fallecidos como consecuencia de la operación; cuatro $(5,4 \%)$ pacientes del grupo A y dos $(1,9 \%)$ del grupo B ( $<<0,05)$. Las causas en el grupo A fueron: absceso subfrénico en 2 y filtración de anastomosis esófago-yeyunal en 2. En el grupo B fueron: shock séptico asociado a íleo mecánico en uno y filtración del muñón duodenal en otro.

La sobrevida estimada a 5 años de los enfermos sometidos a una GE fue de 31,4\% y la de los enfermos sometidos a una GNE de 50,9\%.

El análisis bivariado mostró que el sexo masculino, los mayores de 65 años, la GE, el nivel de albúmina menor a $3,5 \mathrm{~g} / \mathrm{dL}$, el recuento disminuido de linfocitos y la reoperación fueron factores pronósticos asociados a una mayor morbilidad y mortalidad (Tabla 4). El análisis multivariado ajustado por edad y sexo, mostró que un nivel de albúmina preoperatorio menor a 3,5 g/dL y la GE se asociaron a una mayor morbilidad y mortalidad postoperatoria (Tabla 5).

\section{Discusión}

La comparación de los resultados de la GE entre diferentes estudios es difícil de realizar porque existen múltiples definiciones sobre este procedimiento quirúrgico. Algunos la definen como aquella gastrectomía que incluye la resección de un órgano adyacente al estómago bajo la sospecha de estar comprometido por tumor con el fin de realizar una resección oncológica completa y otros la definen de acuerdo al nivel del compromiso y extensión de la disección ganglionar realizada ${ }^{10-12}$. Sin embargo, la mayoría de las comunicaciones utilizan una mezcla de las dos definiciones. En este estudio se utilizó la primera definición y se consideró a la disección ganglionar D2 como parte del tratamiento estándar del cáncer gástrico.

En esta comunicación se observó que los enfermos sometidos a una GE presentaron un mayor porcentaje de complicaciones postoperatorias, complicaciones postoperatorias graves y una mayor mortalidad que aquellos enfermos sometidos a una GNE. También se observó que $71 \%$ de los enfermos sometidos a una GE presentaron un tumor en estadio III ó IV (casi 50\% en estadio IV), confirmando que estos enfermos presentan tumores más avanzados y una situación oncológica de mal pronóstico.

La decisión de practicar una GE se debe fundamentar en el análisis intraoperatorio de múltiples variables. Como concepto general, se debe lograr un equilibrio entre la posibilidad de realizar una resección tumoral completa con la eventual morbilidad y mortalidad asociada. Debido a que la toma de decisiones es generalmente intraoperatoria, esta decisión debe ser individualizada tomando en consideración la envergadura de la resección 
visceral, la complejidad de las reconstrucciones digestivas y las comorbilidades del paciente. Cuando el riesgo teórico perioperatorio se estime mayor al potencial beneficio de una resección extendida no parece razonable su realización. Sin embargo, esta estimación de riesgo es difícil de objetivar. La asociación entre el volumen quirúrgico y los resultados perioperatorios, podrían ayudar a definir esta situación. También sería importante evaluar la calidad de vida de los enfermos tratados sin resección y la posibilidad que ellos tienen para lograr un tratamiento paliativo de buena calidad.

Varios estudios han evaluado las complicaciones y la mortalidad de la GE al analizar aquellos enfermos tratados con una resección multi-orgánica y/o disección ganglionar extendida ${ }^{6-13}$. Con el objetivo de obtener factores pronósticos de complicaciones postoperatorias y mortalidad, Viste y cols ${ }^{17}$ evaluaron los resultados quirúrgicos de 1.010 enfermos sometidos a una gastrectomía y observaron $28 \%$ de morbilidad y $6 \%$ de mortalidad. Al igual que en este estudio, las complicaciones más frecuentes fueron las de origen pulmonar y cardíaco. El género masculino, la edad, el tipo de procedimiento, el uso de antibióticos y la esplenectomía fueron predictores de complicaciones, mientras que el género y la edad fueron predictores de mortalidad. Posteriormente, Bonenkamp et $\mathrm{al}^{18}$, en un estudio prospectivo y randomizado tendiente a dilucidar los beneficios en sobrevida de la disección ganglionar D1 vs D2, observaron que la morbilidad de la gastrectomía asociada a esplenectomía fue de $41 \%$, llegando a $55 \%$ cuando se asoció a una pancreatectomía distal y esplenectomía. En otro estudio, Csendes et a ${ }^{19}$ comunicaron que los pacientes en que se asoció una esplenectomía a la resección gástrica estándar presentaron más complicaciones postoperatorias, pero de una cuantía mucho menor a lo comunicado por Bonenkamp. Casi simultáneamente, Martin et al analizaron los resultados de 1.244 pacientes sometidos a una gastrectomía por cáncer gástrico, asociada o no a la resección de otro órgano y estratificaron las complicaciones de acuerdo a parámetros predefinidos y previamente validados. En el análisis general, no observaron diferencias en las complicaciones ni en la mortalidad entre los dos grupos. Sin embargo, en el análisis específico observaron un mayor número de complicaciones y de complicaciones graves en el subgrupo de enfermos sometidos a una pancreatectomía distal y esplenectomía. También observaron que a medida que aumentaba el número de órganos resecados aumentaban las complicaciones y la estadía hospitalaria. Los factores pronósticos asociados a morbilidad fueron la edad mayor de 70 años y el número de órganos resecados. En este estudio, se utilizó la misma clasificación y estratificación de las complicaciones postoperatorias propuesta por Martin. La estratificación de complicaciones permite comparar objetivamente lo reportado en diferentes publicaciones. Destaca el hecho de que la mayoría de las complicaciones reportadas en esta comunicación corresponden a los grados 1 y 2 , que requieren de manejo específico de baja complejidad y no se asociaron a mortalidad. Las complicaciones grado 3 y 4 se asociaron a mortalidad perioperatoria. Además, la mortalidad global y la asociada a GE de esta serie fueron comparables a lo reportado por centros focalizados en el tratamiento de esta patología ${ }^{20}$. Específicamente, llama la atención en este estudio el bajo porcentaje de fístulas de la unión esofagoyeyunal, que es inferior a lo previamente comunicado ${ }^{1,13,17,19}$. Sólo dos pacientes fueron diagnosticados con esta complicación, que en ellos se asoció a un cuadro séptico que requirió una reoperación y finalmente fallecieron. Por una parte podría estar asociado a una baja detección, debido que sólo un grupo seleccionados de pacientes fueron estudiados en forma específica y algunas fístulas sin relevancia clínica podrían no haber sido diagnosticadas. Es difícil de determinar si la detección de fistulas sin relevancia clínica tendría algún beneficio para el paciente y se asociarían a un cambio en el tratamiento final. Por otra parte, también podría estar asociado a la técnica quirúrgica, que incluyó la cobertura de la anastomosis mecánica con un segundo plano. Para obtener una conclusión más certera en relación a este último punto específico es necesario un estudio prospectivo y randomizado.

Es indudable que se debería determinar si un procedimiento eventualmente riesgoso se justifica en terminos de calidad de vida y sobrevida. No hay datos concluyentes que comparen la calidad de vida y la sobrevida de aquellos pacientes en estadios tumorales avanzados tratados con una gastrectomía extendida frente a aquellos pacientes tratados con observación o quimioterapia. Esto sólo se podría evaluar con un estudio prospectivo y randomizado que compare enfermos tratados con GE con aquellos tratados sin resección. 
Varios estudios han mostrado que la sobrevida estimada a 5 años en los enfermos sometidos a este procedimiento sería entre $16 \%$ y $32 \%$, con una mediana entre 11 y 32 meses $^{8,21}$. En este estudio se observó que la sobrevida estimada a 5 años de los enfermos tratados con una GE fue de $31,4 \%$. Sin embargo, la comparación entre procedimientos y sus resultados tiene el sesgo de la inclusión de pacientes en diferentes estadios tumorales, y que no todos los pacientes resecados presentaban finalmente compromiso del órgano adyacente resecado. En este estudio se observó que el compromiso tumoral del órgano adyacente siempre estuvo presente en aquellos enfermos con invasión del esófago y del diafragma, fue frecuente en los pacientes con compromiso del colon o páncreas y estuvo ausente en el hígado y el bazo. Lamentablemente, en ocasiones es muy difícil definir con certeza la invasión de los órganos adyacentes durante una operación, y esta sospecha finalmente puede representar desde inflamación hasta franca infiltración tumoral. Debido a que la resección oncológica requiere de cirugía regional en block sin contaminación tumoral del lecho quirúrgico, la resección multivisceral debe realizarse sin objetivar previamente la supuesta invasión. El informe anátomo patológico final dilucidará la invasión y por ende el estadío.

Podemos concluir de este estudio, que los enfermos sometidos a una GE, presentaron más complicaciones generales, complicaciones más graves y mayor mortalidad que aquellos enfermos sometidos a una GNE. Sin embargo, la GE se justificaría si las condiciones locales permiten realizar una resección oncológica completa, el paciente no presenta comorbilidades graves y el equipo quirúrgico está en condiciones de ofrecer la cirugía requerida con resultados adecuados.

\section{Referencias}

1. García C, Benavides C, Apablaza S, Rubilar P, Covacevich S, Peñaloza P, et al. [Surgical treatment of gastric cancer: results in 423 cases]. Rev Med Chile 2007; 135: 687-95.

2. Heise K, Bertran E, Andia M, Ferreccio C. Incidence and survival of stomach cancer in a high-risk population of Chile. World J Gastroenterol 2009; 15: 1854-62.

3. Ministerio de Salud de Chile. Obtenido de: http://deis. minsal.cl/deis/ind_2007.asp.

4. Csendes A, Smok G, Medina E, Salgado I, Rivera R, Quitral M. [Clinical course characteristics of gastric cancer
1958-1990]. Rev Med Chile 1992; 120: 36-42.

5. Siewert J, Böttcher K, Stein H, Roder J. Relevant prognostic factors in gastric cancer: ten-year results of the German Gastric Cancer Study. Ann Surg 1998; 228: 449-61.

6. Ozer I, Bostanci E, Orug T, Ozogul Y, Ulas M, Ercan $\mathrm{M}$, et al. Surgical outcomes and survival after multiorgan resection for locally advanced gastric cancer. Am J Surg 2009; 198: 25-30.

7. Kim J, Jang Y, Park S, Park S, Kim S, Mok Y, et al. Surgical outcomes and prognostic factors for T4 gastric cancers. Asian J Surg 2009; 32: 198-204.

8. Martin R, Jaques D, Brennan M, Karpeh M. Extended local resection for advanced gastric cancer: increased survival increased morbidity. Ann Surg 2002; 236: 15965.

9. Jeong O, Choi W, Park Y. Appropriate selection of patients for combined organ resection in cases of gastric carcinoma invading adjacent organs. J Surg Oncol 2009; 100: 115-20.

10. Saito H, Tsujitani S, Maeda Y, Fukuda K, Yamaguchi $\mathrm{K}$, Ikeguchi $\mathrm{M}$, et al. Combined resection of invaded organs in patients with T4 gastric carcinoma. Gastric Cancer 2001; 4: 206-11.

11. Saidi R, Remine S, Dudrick P, Hanna N. Is there a role for palliative gastrectomy in patients with stage IV gastric cancer? World J Surg 2006; 30: 21-7.

12. Carboni F, Lepiane P, Santoro R, Lorusso R, Mancini $\mathrm{P}$, Sperduti I, et al. Extended multiorgan resection for T4 gastric carcinoma: 25-year experience. J Surg Oncol 2005; 90: 95-100.

13. Martin R, Jaques D, Brennan M, Karpeh M. Achieving RO resection for locally advanced gastric cancer: is it worth the risk of multiorgan resection?. J Am Coll Surg 2002; 194: 568-77.

14. Butte J, Becker F, Visscher A, Waugh E, Meneses M, Court I, et al. [Gastroesophageal junction tumors. Early results, long-term survival and prognostic factors in resected patients]. Rev Med Chile 2010; 138: 62-9.

15. Japanese Gastric Cancer Association. Japanese classification of gastric carcinoma--2nd English edition--response assessment of chemotherapy and radiotherapy for gastric carcinoma: clinical criteria. Gastric Cancer 2001; 4: $1-8$.

16. American Cancer Society. American Joint Committee on Cancer. AJCC Cancer Staging Manual. $6^{\text {th }}$ Edition. Lippincott-Raven. 2002.

17. Viste A, Haùgstvedt T, Eide G, Søreide O. Postoperative complications and mortality after surgery for gastric cancer. Ann Surg 1988; 207: 7-13.

18. Bonenkamp J, Hermans J, Sasako M, Van De Velde 
C, Welvaart K, Songun I, et al. Extended lymph-node dissection for gastric cancer. N Engl J Med 1999; 340: 908-14.

19. Csendes A, Burdiles P, Rojas J, Braghetto I, Díaz J, Maluenda F. A prospective randomized study comparing D2 total gastrectomy versus D2 total gastrectomy plus splenectomy in 187 patients with gastric carcinoma. Surgery 2002; 131: 401-7.
20. Adachi Y, Kitano S, Sugimachi K. Surgery for gastric cancer: 10-year experience worldwide. Gastric Cancer 2001; 4: 166-74.

21. Yamamoto M, Baba H, Kakeji Y, Endo K, Ikeda Y, Toh $\mathrm{Y}$, et al. Postoperative morbidity/mortality and survival rates after total gastrectomy, with splenectomy/pancreaticosplenectomy for patients with advanced gastric cancer. Hepatogastroenterology 2004; 51: 298-302. 